\section{Delayed diagnosis of HIV infection in ophthalmic practice}

AC Wadood', B Dhillon ${ }^{1,2}$, G Mcllwaine ${ }^{1,3}$ and RP Brettle ${ }^{2}$

\begin{abstract}
The introduction of potent antiretroviral drug combinations has transformed human immunodeficiency virus/acquired immunodeficiency disease syndrome (HIV/ AIDS) from an intractable and rapidly fatal disease to a chronic manageable illness with prolonged life survival for many patients. This paper discusses the ophthalmic and medical histories of two young female patients who presented with 'atypical' optic neuropathy and toxoplasma gondii retinochoroiditis and were later found to be HIV infected. We discuss the need for increased vigilance in the ophthalmic community for suspecting HIV infection to allow optimal management of the ophthalmic and systemic manifestations of the disease spectrum associated with HIV/AIDS.
\end{abstract}

Eye (2004) 18, 293-298. doi:10.1038/

sj.eye. 6700630

Keywords: HIV infection; optic neuritis; toxoplasma retinochoroiditis

\section{Case 1}

A 25-year-old female presented in September 1996 with a 1-day history of painful visual loss in the left eye (LE). Examination of the LE showed unaided visual acuity of $6 / 60$ improving to $6 / 18$ with correction, left relative afferent pupillary defect (RAPD), and pain on ocular movements. The clinical findings were consistent with the diagnosis of left retrobulbar optic neuritis. After 1 week, the visual acuity decreased to no perception of light in the LE, with marked RAPD and optic nerve head swelling. During this period, she developed blurring of vision (visual acuity, 6/9) in the right eye (RE), with occasional retro-orbital pain. The magnetic resonance imaging (MRI) carried out revealed an area of high signal within the left optic nerve, two areas of high signal in the parietal and paratrigonal white matter, and a possible abnormal area in the anterior corpus callosum on the right. The presence of these three lesions made the demyelination a distinct possibility. Cerebrospinal fluid examination was normal with no oligoclonal bands detected. Leber's genetic screening was negative. Based on the clinical and radiological findings, she was treated initially with intravenous methyl prednisolone and then a reducing dose of oral steroids. The examination of the RE was unremarkable.

With no improvement in her condition, a combination therapy with azathioprine and low-dose systemic steroid was recommended in June 1997, nearly 9 months after the onset of optic neuritis. She developed neutropenia; consequently, the azathioprine was discontinued. However, the neutropenia persisted even after stopping the drug. During this period, she had intermittent episodes of fatigue and breathlessness and chest radiograph suggested sarcoidosis-like changes. In the following years, she had episodes of nonspecific urethritis and herpetic genital infections.

Her current medicines included systemic antibiotics for urinary tract infection (UTI) and oral contraceptives, which she started at the age of 15 years. Past medical history included recurrent UTI and genital infections with herpes simplex and thrush. Two of her previous sexual contacts (one 4 years ago and the other 10 years ago) later turned out to be HIV-positive. HIV testing was prompted in early 2000, when she became aware of the fact that one of her previous partners had become HIV-positive. On testing, she was found to be positive for HIV infection and accordingly started on a combination antiretroviral therapy. Since the diagnosis of HIV infection, this patient's general neurological function has deteriorated with sensory loss of right arm and leg, but the eye condition remains unchanged to the present day.

\section{Case 2}

A 34-year-old female presented in August 2001, complaining of pain, photophobia, and redness
${ }^{1}$ Princess Alexandra Eye Pavilion

Edinburgh, UK

${ }^{2}$ Regional Infectious

Diseases Unit

Edinburgh, UK

${ }^{3}$ Department of Clinical Neurosciences Lothian University Hospitals Edinburgh, UK

Correspondence: AC Wadood Princess Alexandra Eye Pavilion

Chalmers Street

Royal Infirmary Edinburgh Edinburgh, UK

Tel: +4401315361000 Fax: + 4401315364090

E-mail: azfarwadood@ aol.com

Received: 28 June 2002 Accepted: 24 April 2003

None of the authors or department has any proprietary or commercial interest related to the products or instruments described in this article

Presented at the Scottish Ophthalmological Club Edinburgh, March 2002 


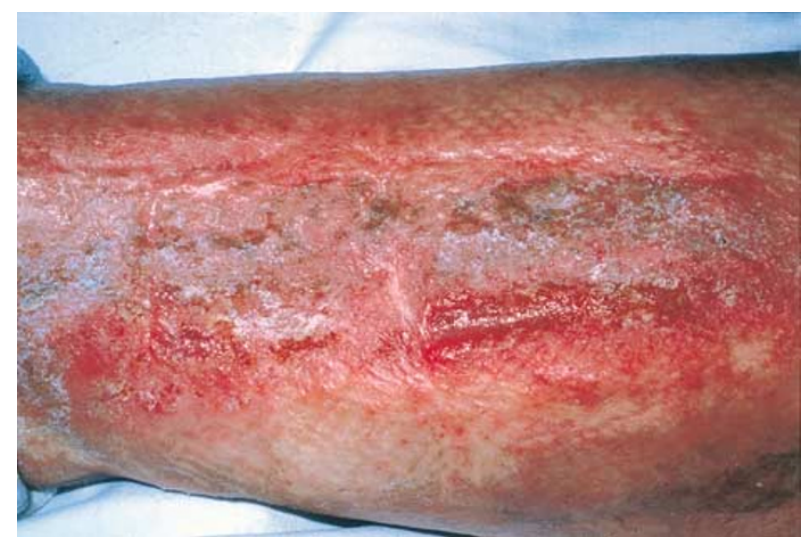

Figure 1 Necrotising fasciitis of the leg following minor trauma in patient 2 .

with deterioration of vision in her RE of 6 days duration. The previous general history in this case was complicated and began with her developing immune thrombocytopenic purpura (ITP) in 1989. ITP was treated initially with immunoglobulins and systemic steroids without success. She eventually had splenectomy for uncontrolled ITP, 12 years previously. At this stage, she developed psychiatric symptoms and was diagnosed to have depressive maniac disorder. Since the splenectomy, she has had recurrent episodes of pneumonia, regular oral and genital thrush, several courses of cryotherapy for cervical dysplasia, auditory infection with reduced hearing, intermittent migraine, peptic ulcer, and seborrhic dermatitis. She developed necrotising fasciitis and septicaemia following minor trauma to her leg in October 2000 (Figure 1). This required blood transfusions and several autologous skin grafts. There was no past ocular history of any significance. Her current medication included long-term penicillin and fluconazole for thrush.

On presentation, she had best-corrected visual acuity (BCVA) of 6/12 in the RE. Slit-lamp examination revealed ciliary injection, $3+$ cells in the anterior chamber with posterior synechae. The dilated fundus examination of the RE showed grade II vitritis with a slightly raised creamy white exudative lesion, in the superotemporal retinal quadrant, findings consistent with toxoplasma retinochoroiditis (Figure 2, top). There was no associated old chorioretinal scar in the affected eye. Examination of the LE was unremarkable.

Investigations including blood cultures, serum $\mathrm{ACE}$, serum $\mathrm{Ca}^{2+}, \mathrm{ESR}$, all were within normal limits with FBC showing lymphopenia (0.90, normal range 1.5-4.0 $\times$ $\left.10^{9} / 1\right)$. The serum for Toxoplasma gondii IgG antibody was positive with IgM antibodies not detectable. Polymerase chain reaction for toxoplasma was negative in the aqueous humour sample. Computed tomography (CT)
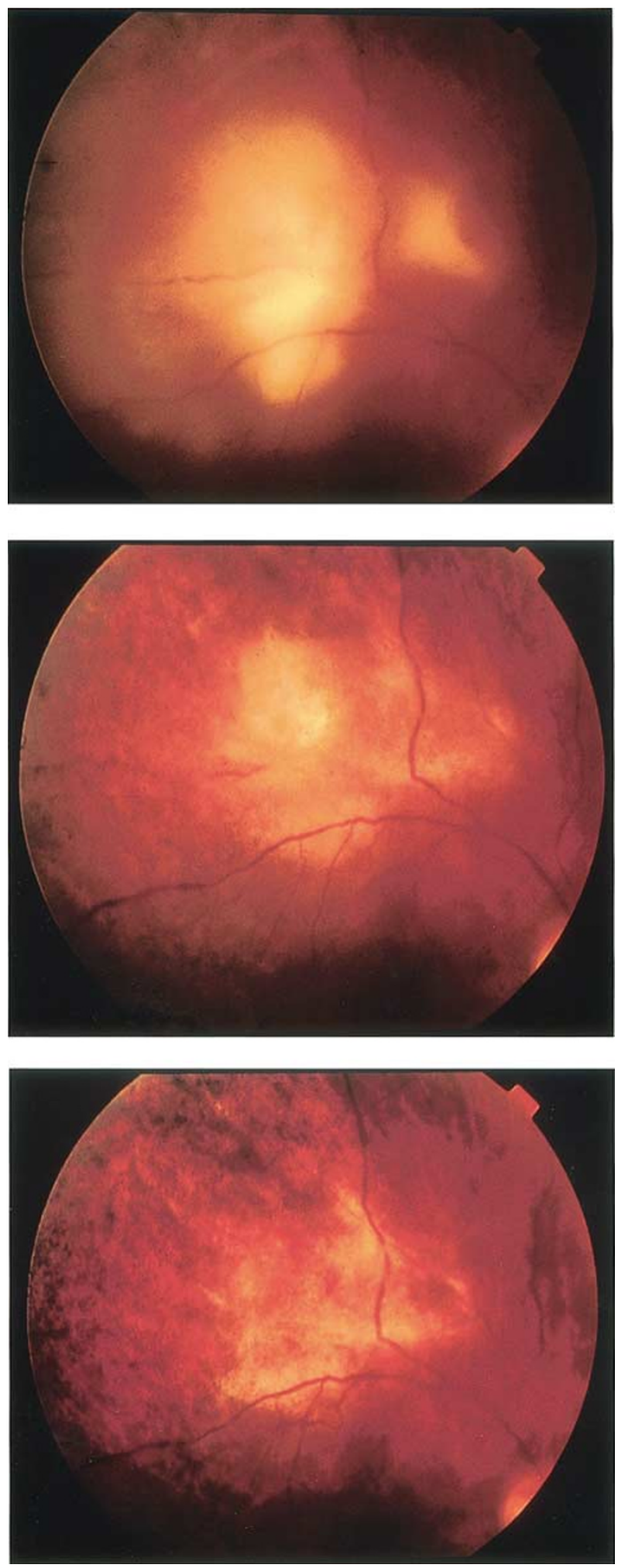

Figure 2 Toxoplasma retinochoroiditis in the superotemporal retinal quadrant of the right eye at presentation (top). Lesion showing signs of regression at 1- (middle) and 3-week (bottom) follow-up. 

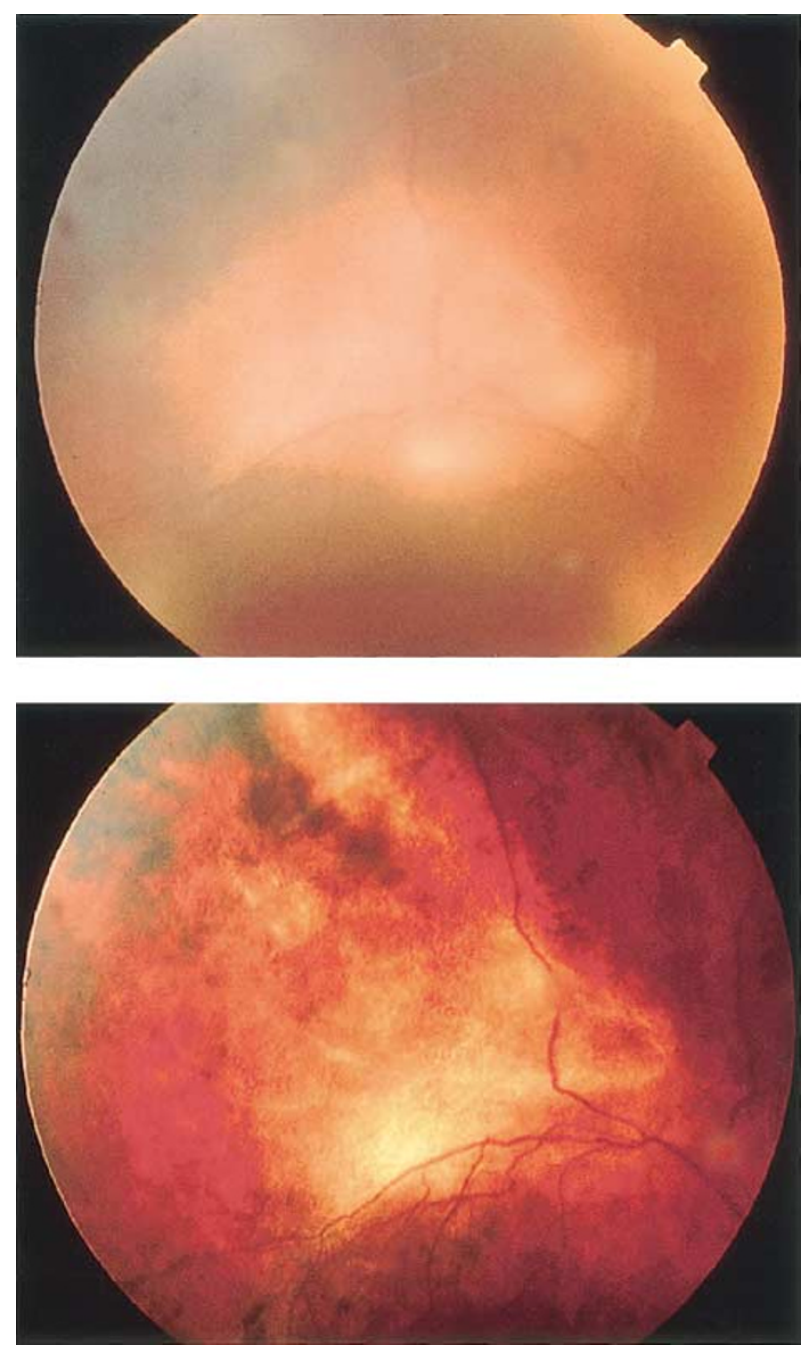

Figure 3 Reactivation of the retinochoroiditis 6 weeks later (top). Same lesion showing signs of improvement on antiretroviral and antitoxoplasma therapy (bottom).

excluded the presence of intracranial toxoplasma cysts. She was commenced on antitoxoplasma therapy comprising of oral sulphadiazine $1 \mathrm{~g}$ q.d.s., pyrimethamine $25 \mathrm{mg}$ twice a day and folinic acid $15 \mathrm{mg}$ twice a week, along with topical steroids. After 4 days, there was further deterioration in the BCVA $(6 / 60)$ in the affected eye, and systemic prednisolone $20 \mathrm{mg} /$ day added to the antitoxoplasma therapy. While on the current medication, she developed an episode of 'unconsciousness' and psychotic symptoms and stopped all her medications including antiparasitic therapy. When examined at 1 week and 3 weeks off the systemic treatment, this lady was generally feeling better, visual acuity improving to $6 / 18$ in the RE and the fundal lesion showing signs of regression (Figure 2, middle and bottom, respectively). Systemic therapy was not recommenced because of the improvement in ocular and systemic condition and the possibility of steroid-induced psychosis.

After 3 weeks, she presented again with 2-day history of increasing pain and reduced visual acuity in her RE with recurrence of the active retinochoroiditis (Figure 3, top). The reactivation of retinochoroiditis urged further investigations, which led to the diagnosis of HIV infection in November 2001. Antitoxoplasma triple therapy as mentioned above was recommenced. After 2 weeks of treatment, sulphadiazine had to be changed to clindamycin $450 \mathrm{mg}$ q.d.s, as the former became unobtainable owing to problems with the manufacturing. The fundal lesion showed signs of improvement after 8 weeks of antiretroviral and antitoxoplasma therapy (Figure 3, bottom). Antitoxoplasma therapy was discontinued after 6 months, as the retinochoroiditis remained quiescent. The improvement in the fundal lesion was paralleled by an improvement in the immune function as assessed by CD4 T-lymphocyte count, 65 cells $/ \mathrm{mm}^{3}$ at the time of diagnosis of HIV infection, 225 cells $/ \mathrm{mm}^{3}$ after 4 weeks of antiretroviral treatment, to 332 cells $/ \mathrm{mm}^{3}$ when antitoxoplasma therapy was stopped.

\section{Discussion}

The clinical course of these two patients highlights the markedly heterogeneous ocular and systemic manifestations in patients with HIV infection. There was a delay of several years in the current cases before reaching the actual diagnosis, explaining the multiplicity of the medical problems that these young patients had over the years. To avoid such a delay, universal HIV testing with active patient refusal is recommended for areas of high HIV prevalence. More can be learnt from the experience of HIV testing in the antenatal care. HIV testing should be offered to the patients presenting from the high-risk group (homosexuals, intravenous drug addicts, haemophiliacs, heterosexuals), those with atypical and/or relapsing ophthalmic disease, and requiring immunosuppression or are already immunosuppressed.

Counselling is provided routinely before and after the HIV antibody testing in HIV/AIDS care units. Stigma and fear associated with the diagnosis of HIV/AIDS can be minimised if patients are reassured that their diagnosis and details are going to be dealt with sensitively and confidentially. Before proceeding with the HIV testing, patients should be informed that this is part of their routine care, given ample time to consider the timing of HIV test and consider potential risk factors for HIV infection. Timing of the test is important, as the patient may not be prepared adequately, although on the other hand, seroconversion may have not occurred and 
therefore the test may not represent the HIV status. Patients should be fully informed about the implications should they test positive, and how this can be managed, with ongoing support and the possibility of medication. Patients should be asked to return in person for the test result. This maintains the confidentiality and provides opportunity to discuss issues surrounding a positive HIV test. If the test is reported negative, this provides an opportunity to inform people about the risk of other infections, or more about the HIV and safer sex practices.

HIV-infected adults may experience a diverse pattern and time-course of progression of their illness.

Seroconversion with the production of antibodies usually occurs between 2 and 6 weeks following HIV infection, it may take up to 12 weeks. A proportion of infected individuals (up to $80 \%$ ) may experience a self-limiting 'seroconversion illness', lasting for about 2-3 weeks. ${ }^{1}$ The usual manifestations of this illness are mild and their significance overlooked: headache, fever,

lymphadenopathy, sore throat, and a rash.,3

Occasionally, the illness may have quite severe neurological symptoms of meningitis, encephalitis, ${ }^{4}$ myelitis, and Guillain-Barré syndrome, which all seem to resolve spontaneously. ITP is a well-described complication of HIV infection, which occurs equally frequently during the early asymptomatic phase of HIV infection and with the clinical AIDS. ITP has been reported to occur in 10-40\% of HIV-infected patients at different stages of the disease. ${ }^{5,6}$ One study ${ }^{7}$ showed that it takes an average of 10 years from infection to death or developing AIDS.

Ophthalmologists should be aware of the general as well as ocular manifestations of HIV infection according to the stage of the disease. At the seroconversion phase, injection of conjunctiva, headache, and retro-orbital pain may be experienced. In the early-stage Sjogren's syndrome, allergic conjunctivitis, Reiter's syndrome, HIV retinopathy, intermediate uveitis, retinal vasculitis, and optic neuropathy may occur. The intermediate stage of HIV infection may be associated with dry eye syndrome, blepharitis, conjunctivitis, molluscum contagiosum, Kaposi's sarcoma, herpes zoster, herpes simplex infections, HIV retinopathy, tuberculous uveitis, orbital aspergillosis, and optic neuropathy.

Survival and quality of life has improved substantially for people with HIV infection over the recent years. The main advances have been in the use of plasma HIV quantification ('viral load') and the availability of new antiretroviral drugs, the protease inhibitors. CD4 Tlymphocyte number in the blood samples have provided a clinically useful marker of the level of immunodeficiency in an individual at a given time point. ${ }^{8,9} \mathrm{CD} 4 \mathrm{~T}$-lymphocyte counts in the blood persistently below a value of 500 cells $/ \mathrm{mm}^{3}$ is considered abnormal. CD4 T-lymphocyte counts may be low because of the cells leaving the circulation and entering the lymph nodes in numerous other infective illnesses such as TB, and common viral infections. In these cases, when unassociated with HIV, the percentage of CD4 cells to total T-cell number is preserved, as is the ratio of CD4 to CD8 T cells. It is usually only when CD4 counts fall below 200 cells $/ \mathrm{mm}^{3}$ that patients become vulnerable to the more severe and AIDS-defining opportunistic infections.

Neuro-ophthalmic abnormalities may present to ophthalmologists and may be the presenting feature of different stages of the HIV infection. One clinical survey showed that neurological disorders occur in $40 \%$ of adult AIDS patients, ${ }^{10}$ while post-mortem studies reveal neuropathology in 70-80\% of AIDS patients. ${ }^{11}$ Of those with neurological manifestations, $41 \%$ have opportunistic infections. ${ }^{12}$ The organisms most frequently involved are toxoplasma, cryptococcus, cytomegalovirus (CMV), and herpes zoster. HIV may be associated with a large variety of neuro-

ophthalmological manifestations, including optic nerve disease (oedema, inflammation, atrophy), retrobulbar neuritis, visual field defects, cortical blindness, pupillary defects, and ocular motor nerve palsies. Most of these disorders are caused by infectious lesions of the central nervous system.

The clinical syndrome of optic neuritis may occur from involvement by a number of different disease processes including CMV neuroretinits, cryptococcal optic neuropathy, syphilitic perineuritis, hepatitis B or Cinduced optic neuropathy, and optic neuritis associated with herpetic retinitis. There is also a form of optic neuritis that presents as demyelinating optic neuritis with visual loss, abnormal colour vision, pain on eye movement, and a normal fundus. Both optic nerves are affected. The presentation, clinical course and neuropathology are similar to that of optic neuritis of multiple sclerosis (MS). The benefit of systemic steroids for this form of optic neuritis remains uncertain at this time.

There may be a correlation between MS and HIV infection, which is more than a chance occurrence of the two conditions. Berger et al ${ }^{13}$ reported that seven patients with 'definite' MS and HIV disease have been described and in four of these patients, the MS predated the HIV infection by 3.5-18 years. In all these patients, MS progressed rapidly and a majority had optic neuritis. In patients with optic neuritis and rapidly deteriorating neurological condition with an MRI scan compatible with MS, HIV testing should be considered.

There is evidence that the HIV itself can produce an optic neuropathy. There are few reported cases of 
neuropathy apparently caused by direct HIV infection of the nerve, but in all cases, this has been a diagnosis of exclusion. Axonal loss has been reported in optic nerves of AIDS patients without secondary infection. Mean axonal diameter was unchanged indicating that all classes of axon were affected and the degeneration affected the optic nerve diffusely. ${ }^{14}$ It is possible that this nerve fibre loss is a consequence of HIV retinal microvasculopathy with multiple cotton wool spots. Alternately, the degenerative process could be occurring in the optic nerve itself. A study, ${ }^{15}$ with light microscopy showed thickened meninges, axonal degeneration with various stages occurring in each optic nerve, degeneration of oligodendrocytes, and activated microglia. Cases have been reported where all the recognised causes of optic neuropathy in HIV-infected patients were excluded. ${ }^{16}$

Toxoplasmosis in the immunocompromised host is most probably reactivation of a previous latent infection, ${ }^{17}$ although in certain circumstances (leukaemia and organ transplantation), infection can be acquired from blood transfusions and contaminated donor tissue. Acute lesions rarely are associated with pre-existing chorioretinal scars, suggesting newly acquired infection or new dissemination from nonocular sites of infection.

Toxoplasma retinochoroiditis is an infrequent opportunistic infection of the eye in HIV/AIDS patients accounting for $1-3 \%$ of retinal infections seen in AIDS patients. ${ }^{18}$ Ocular toxoplasmosis in AIDS patients is usually unilateral and often, but not invariably, associated with significant anterior uveitis and vitritis, ${ }^{19}$ as noted in the current case. The retinal lesions may be single, multifocal, or diffuse (mimicking acute retinal necrosis) and not associated with or adjacent to an old chorioretinal scar. ${ }^{20}$ Retinal haemorrhages in ocular toxoplasmosis retinochoroiditis are minimal or absent and rhegmatogenous retinal detachment may occur, but is much less frequent than in viral retinitis. Toxoplasma gondii appears to have a predilection for the central nervous system, which is the most common site of involvement in severely immunocompromised hosts. ${ }^{21}$ The reported prevalence of cerebral toxoplasmosis (CTP) in immunosuppressed patients with ocular toxoplasmosis ranges from 20 to $80 \% .{ }^{19,22-24}$ Owing to the greater sensitivity, MRI may be the neuroimaging of choice for the initial radiological evaluation of AIDS patients with neurological illness. However, CT scan of the brain may show single or multiple ring-enhancing lesions in CTP. A similar appearance on CT scan may occur with cerebral lymphoma, and only a stereotactic brain biopsy may be able to differentiate between the two. Certain features are suggestive of CTP as the lesions tend to be multiple, show ring enhancement, and there is marked surrounding oedema with a mass effect. CTP lesions are usually found in the basal ganglion, frontal lobe, parietal lobe, and occipital lobe; on the other hand, lymphomas are often periventricular in position and spread into the ventricle.

Highly destructive and fulminant forms of ocular toxoplasmosis have been reported in patients immunosuppressed by AIDS, ${ }^{19,20,22-25}$ lymphoma and haematologic cancers, ${ }^{23,26-28}$ connective tissue disease, ${ }^{23,28}$ organ and bone marrow transplantation, ${ }^{19,20,23-28}$ and chronic immunosuppressive drug therapy, including chronic corticosteroid treatment. ${ }^{23,26,29,30}$ Toxoplasmosis as a cause of multifocal or diffuse necrotising retinitis or both clinically appearing similar to acute retinal necrosis has been reported in elderly otherwise immunocompetent patients. ${ }^{31}$ Of the importance to ophthalmologists is the fact that ocular disease may be the first manifestation of life-threatening multisystem toxoplasma infection in patients who are immunodeficient. ${ }^{22}$

Most cases of toxoplasma retinitis in patients with AIDS respond to standard antitoxoplasma therapy within 4-6 weeks, but also require long-term maintenance dose to prevent relapse. The widespread use of oral cotrimoxazole as a primary prophylaxis for Pneumocystitis carinii pneumonia appears to have decreased the frequency of ocular toxoplasmosis in patients with AIDS. ${ }^{32}$

Ophthalmic consultation is often to rule out the AIDS defining ocular condition in an already diagnosed HIV infected patient. This paper stresses the need for increased vigilance on part of the ophthalmologists for suspecting HIV infection to allow optimal management of patients presenting with the eye disease. Testing patients for HIV is but carried out by personnel trained in discussing the implications should a positive result emerge.

\section{References}

1 Tindall B, Barker S, Donovan B, Barnes T, Roberts J, Kronenberg $\mathrm{C}$ et al. Characterization of the acute clinical illness associated with human immunodeficiency virus infection. Arch Intern Med 1988; 148(4): 945-949.

2 Tindall B, Cooper DA. Primary HIV infection: host responses and intervention strategies. AIDS 1991; 5(1): 1-14.

3 Fox R, Eldred LJ, Fuchs EJ, Kaslow RA, Visscher BR, Ho M et al. Clinical manifestations of acute infection with human immunodeficiency virus in a cohort of gay men. AIDS 1987; 1(1): 35-38.

4 Hardy WD, Daar ES, Sokolov Jr RT, Ho DD. Acute neurologic deterioration in a young man. Rev Infect Dis 1991; 13(4): 745-750.

5 Sloand EM, Klein HG, Banks SM, Vareldzis B, Merritt S, Pierce P. Epidemiology of thrombocytopenia in HIV infection. Eur J Haematol 1992; 48(3): 168-172.

6 Sullivan PS, Hanson DL, Chu SY, Jones JL, Ciesielski CA and the Adult/Adolescent Spectrum of Disease Group. 
Surveillance for thrombocytopenia in persons infected with HIV: results from the multistate adult and adolescent spectrum of disease project. J Acquir Immune Defic Syndr Hum Retrovirol 1997; 14: 374-379.

7 Rutherford GW, Lifson AR, Hessol NA, Darrow WW, O'Malley PM, Buchbinder SP et al. Course of HIV-I infection in a cohort of homosexual and bisexual men: an 11 year follow up study. BMJ 1990; 301(6762): 1183-1188.

8 Goedert JJ, Biggar RJ, Melbye M, Mann DL, Wilson S, Gail MH et al. Effect of T4 count and cofactors on the incidence of AIDS in homosexual men infected with human immunodeficiency virus. JAMA 1987; 257(3): 331-334.

9 Polk BF, Fox R, Brookmeyer R, Kanchanaraksa S, Kaslow R, Visscher B et al. Predictors of the acquired immunodeficiency syndrome developing in a cohort of seropositive homosexual men. $N$ Engl J Med 1988; 362: 61-66.

10 Levy RM, Bredesen DE, Rosenblum ML. Neurological manifestations of the acquired immunodeficiency syndrome (AIDS): experience at UCSF and review of the literature. J Neurosurg 1985; 62(4): 475-495.

11 Petito CK, Cho ES, Lemann W, Navia BA, Price RW. Neuropathology of acquired immunodeficiency syndrome (AIDS): an autopsy review. J Neuropathol Exp Neurol 1986; 45(6): 635-646.

12 Guiloff RJ. AIDS: Neurological opportunistic infection in Central London. J R Soc Med 1989; 82: 278-280.

13 Berger JR, Sheremata WA, Resnick L, Atherton S, Fletcher MA, Norenberg M. Multiple sclerosis-like illness occurring with human immunodeficiency virus infection. Neurology 1989; 39(3): 324-329.

14 Tenhula WN, Xu SZ, Madigan MC, Heller K, Freeman WR, Sadun AA. Morphometric comparisons of optic nerve axon loss in acquired immunodeficiency syndrome. Am J Ophthalmol 1992; 113(1): 14-20.

15 Sadun AA, Pepose JS, Madigan MC, Laycock KA, Tenhula WN, Freeman WR. AIDS-related optic neuropathy: a histological, virological and ultrastructural study. Graefe's Arch Clin Exp Ophthalmol 1995; 233: 387-398.

16 Newman NJ, Lessell S. Bilateral optic neuropathies with remission in 2 HIV positive men. J Clin Neuro-Opthalmol 1992; 12: 1-5.

17 Wong B, Gold JWM, Brown AE, Lange M, Fried R, Grieco M et al. Central-nervous-system toxoplasmosis in homosexual men and parenteral drug abusers. Ann Intern Med 1984; 100: 36-42.
18 Rodgers CA, Harris JR. Ocular toxoplasmosis in HIV infection. Int J STD AIDS 1996; 7(5): 307-309.

19 Cochereau-Massin I, LeHoang P, Lautier-Frau M, Zerdoun $\mathrm{E}$, Zazoun L, Robinet $\mathrm{M}$ et al. Ocular toxoplasmosis in human immunodeficiency virus-infected patients. Am J Ophthalmol 1992; 114(2): 130-135.

20 Gagliuso DJ, Teich SA, Friedman AH, Orellana J. Ocular toxoplasmosis in AIDS patients. Trans Am Ophthalmol Soc 1990; 88: 63-86.

21 Ryning FW, Mills J. Pneumocystis carinii, Toxoplasma gondii, cytomegalovirus and the compromised host. West J Med 1979; 130: 18-34.

22 Holland GN, Engstrom Jr RE, Glasgow BJ, Berger BB, Daniels SA, Sidikaro Y et al. Ocular toxoplasmosis in patients with the acquired immunodeficiency syndrome. Am J Ophthalmol 1988; 106(6): 653-667.

23 Elkins BS, Holland GN, Opremcak EM, Dunn Jr JP, Jabs DA, Johnston WH et al. Ocular toxoplasmosis misdiagnosed as cytomegalovirus retinopathy in immunocompromised patients. Ophthalmology 1994; 101(3): 499-507.

24 Pivetti-Pezzi P, Accorinti M, Tamburi S, Ciapparoni V, Abdulaziz MA. Clinical features of toxoplasmic retinochoroiditis in patients with acquired immunodeficiency syndrome. Ann Ophthalmol 1994; 26 : 73-84.

25 Moorthy RS, Smith RE, Rao NA. Progressive ocular toxoplasmosis in patients with acquired immunodeficiency syndrome. Am J Ophthalmol 1993; 115(6): 742-747.

26 Yeo JH, Jakobiec FA, Iwamoto T, Richard G, Kreissig I. Opportunistic toxoplasmic retinochoroiditis following chemotherapy for systemic lymphoma. A light and electron microscopic study. Ophthalmology 1983; 90(8): 885-898.

27 Hoerni B, Vallat M, Durand M, Pesme D. Ocular toxoplasmosis and Hodgkin's disease: report of two cases. Arch Ophthalmol 1978; 96(1): 62-63.

28 Ruskin J, Remington JS. Toxoplasmosis in the compromised host. Ann Intern Med 1976; 84(2): 193-199.

29 Sabates R, Pruett RC, Brockhurst RJ. Fulminant ocular toxoplasmosis. Am J Ophthalmol 1981; 92(4): 497-503.

30 Nicholson DH, Wolchok EB. Ocular toxoplasmosis in an adult receiving long-term corticosteroid therapy. Arch Ophthalmol 1976; 94(2): 248-254.

31 Johnson MW, Greven GM, Jaffe GJ, Sudhalkar H, Vine AK. Atypical, severe toxoplasmic retinochoroiditis in elderly patients. Ophthalmology 1997; 104(1): 48-57.

32 Jabs DA, Bartlett JG. AIDS and ophthalmology: a period of transition. Am J Ophthalmol 1997; 124(2): 227-233. 